UCRL-PROP-130636

\title{
Solvent Mediated Self-Assembly of Solids
}

\author{
J. De Yoreo \\ W. D. Wilson \\ T. Palmore
}

December 12, 1997

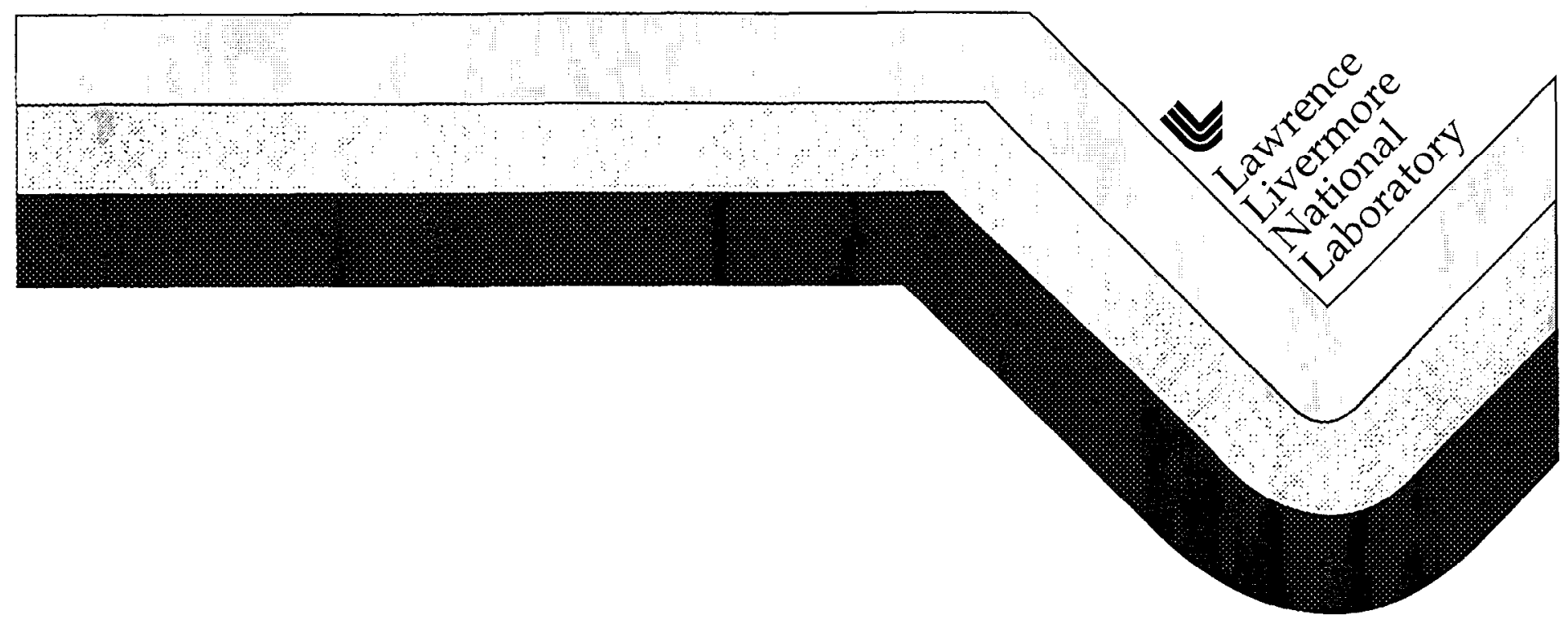




\section{DISCLAIMER}

This document was prepared as an account of work sponsored by an agency of the United States Government. Neither the United States Government nor the University of California nor any of their employees, makes any warranty, express or implied, or assumes any legal liability or responsibility for the accuracy, completeness, or usefulness of any information, apparatus, product, or process disclosed, or represents that its use would not infringe privately owned rights. Reference herein to any specific commercial product, process, or service by trade name, trademark, manufacturer, or otherwise, does not necessarily constitute or imply its endorsement, recommendation, or favoring by the United States Government or the University of California. The views and opinions of authors expressed herein do not necessarily state or reflect those of the United States Government or the University of California, and shall not be used for advertising or product endorsement purposes.

This report has been reproduced directly from the best available copy.

Available to DOE and DOE contractors from the Office of Scientific and Technical Information

P.O. Box 62, Oak Ridge, TN 37831

Prices available from (615) 576-8401, FTS 626-8401

Available to the public from the

National Technical Information Service

U.S. Department of Commerce

5285 Port Royal Rd.,

Springfield, VA 22161 


\title{
Solvent mediated self-assembly of solids
}

\author{
Investigators \\ Jim De Yoreo, P.I. \\ Lawrence Livermore National Laboratory \\ Livermore, CA 94550 \\ W. D. Wilson, Co-P.I. \\ Sandia National Laboratories \\ Livermore, CA 94550 \\ Tayhas Palmore, Co-P.I. \\ Department of Chemistry \\ University of California at Davis \\ Davis, CA 95616
}

\section{A new initiative proposal to the Division of Materials Science, Office of Basic Energy Sciences, Department of Energy}

December 12, 1997

\begin{abstract}
Solvent-mediated crystallization represents a robust approach to self-assembly of nanostructures and microstructures. In organic systems, the relative ease with which the structure of hydrogenbonded molecules can be manipulated allows for generation of a wide variety of nanoscale crystal structures. In living organisms, control over the micron-to-millimeter form of inorganic crystals is achieved through introduction of bio-organic molecules. The purpose of this proposal is to understand the interplay between solution chemistry, molecular structure, surface chemistry, and the processes of nucleation and crystal growth in solvent-mediated systems, with the goal of developing the atomic and molecular basis of a solvent-mediated self-assembly technology. We will achieve this purpose by: 1) utilizing an atomic force microscopy (AFM) approach that provides in situ, real time imaging during growth from solutions, 2) by modifying kinetic Monte Carlo (KMC) models to include solution-surface kinetics, 3) by introducing quantum chemistry (QC) calculations of the potentials of the relevant chemical species and the near-surface structure of the solution, and 4) by utilizing molecular dynamics (MD) simulations to identify the minimum energy pathways to the solid state. Our work will focus on two systems chosen to address both the nanometer and micron-to-millimeter length scales of assembly, the family of 2,5-diketopiperazines (X-DKPs) and the system of $\mathrm{CaCO}_{3}$ with amino acids. Using AFM, we will record the evolution of surface morphology, critical lengths, step speeds, and step-step interactions as a function of supersaturation and temperature. In the case of the X-DKPs, these measurements will be repeated as the molecular structure of the growth unit is varied. In the case of $\mathrm{CaCO}_{3}$, they will be performed as a function of solution chemistry including $\mathrm{pH}$, ionic strength, and amino acid content. In addition, we will measure nucleation rates and orientations of $\mathrm{CaCO}_{3}$ on polyamino acid templates. From these measurements, we will extract fundamental growth parameters for input into KMC simulations whose predictions will in turn be compared to the experimental observations. The KMC simulations will incorporate atomic processes representing the minimum energy pathways as determined from the MD calculations. The interaction potentials of the relevant chemical species as well as the hydrated surface, including the electrochemical double layer, used in the MD simulations will be determined using coupled solutions to the Schrödinger and PoissonBoltzmann equations which take account of electronic relaxation effects.
\end{abstract}




\section{Motivation and Purpose}

Controlling the nanostructure and microstructure of crystalline solids is a major driving force behind materials research. Among the many approaches being explored, self-assembly promises to be one of the most robust, with structure determined at the molecular level. Because crystal growth by its very nature is a rapid, repeatable method of self-assembly occurring in a wide variety of molecular systems both inorganic and organic, it presents an attractive approach to this nanoscale synthesis of solids. A number of crystallization-based avenues to this nascent discipline are being pursued within a broad set of existing disciplines including surface science, 1 organic chemistry, 2-4 and biology. 5-7

Many of the most attractive approaches to self-assembly involve crystallization of solids from aqueous and/or organic solutions. In organic systems, the relative ease with which the structure of hydrogen bonded molecules can be manipulated allows for generation of a wide variety of supramolecular motifs. Establishing control over the size, shape, and the degree of anisotropy of the growth units has been shown to produce well-ordered crystals of molecular tapes, ladders, layers, nano-porous structures, and other motifs for potential application as high density storage media, optoelectronic devices, shape selective catalysts, nano-scale reaction and storage vesicles, and trace-contaminant separators. $2-4$ The basic concept behind this approach to engineering the solid state is that, through control of the structure of the individual molecular units, the final crystal structure is determined by design. Moreover, because hydrogen-bonded molecules can be systematically extended along multiple axes independently, molecular units can be designed to assemble into 1D and 2D aggregates that serve as scaffolds for three dimensional structures.

This approach provides control over the nanometer scale crystal structure. Equally important for self-assembly technologies is the development of control over crystal form and orientation at the micron-to-millimeter scale. By far, the most dramatic examples of tailored molecular self-assembly at this scale are found in naturally occurring mineralized structures generated through biological control over the process of inorganic crystallization. Recent investigations ${ }^{5-9}$ have demonstrated the ability of many living organisms to control crystallization by selective application of bio-organic molecules which enable nucleation and growth of crystalline structures of carbonates, phosphates, oxides, silicates, metals, and other inorganic materials as shown in Table I. By selectively inhibiting or modifying nucleation, step kinetics, surface morphologies, and facet stability, these organisms are able to produce nanophase materials as well as exquisite and topologically complex single-crystals and multi-layer composites, some examples of which are shown in Fig 1 . The resulting materials have functions as diverse as

Table I

Approaches to synthesis of biomineralized structures observed in nature and typical materials produced.

\begin{tabular}{|c|c|c|c|}
\hline Approach & Product & Systems & Materials \\
\hline $\begin{array}{l}\text { Nanoscale } \\
\text { synthesis }\end{array}$ & Clusters, Nanoparticles & $\begin{array}{l}\text { Microemulsions } \\
\text { Vesicles } \\
\text { Ferritin }\end{array}$ & $\begin{array}{l}\mathrm{Pt}, \mathrm{Co}, \text { borides, } \mathrm{CaCO}_{3}, \mathrm{Fe}_{3} \mathrm{O}_{4} \\
\mathrm{ZnS}, \mathrm{Ag}_{2} \mathrm{O}, \mathrm{Al}_{2} \mathrm{O}_{3} \\
\mathrm{UO}_{3}, \mathrm{FeS}, \mathrm{MnOOH}\end{array}$ \\
\hline $\begin{array}{l}\text { Crystal } \\
\text { engineering }\end{array}$ & $\begin{array}{l}\text { Single crystals, } \\
\text { Shaped composites }\end{array}$ & $\begin{array}{l}\text { Monolayers } \\
\text { Protein mixtures } \\
\text { Tubules } \\
\text { Fibres } \\
\text { Polyanionic peptides }\end{array}$ & $\begin{array}{l}\mathrm{CaCO}_{3} \\
\mathrm{NaCl}, \mathrm{CaCO}_{3}, \mathrm{BaSO}_{4} \\
\mathrm{Cu}, \mathrm{Ni}, \mathrm{Al}_{2} \mathrm{O}_{3}, \mathrm{Fe}_{3} \mathrm{O}_{4} \\
\mathrm{CuCl}, \mathrm{CaCO}, \mathrm{Fe}_{2} \mathrm{O}_{3} \\
\mathrm{CaCO}_{3}\end{array}$ \\
\hline $\begin{array}{l}\text { Microstructural } \\
\text { fabrication }\end{array}$ & $\begin{array}{l}\text { Mineral-polymer } \\
\text { composites, } \\
\text { Organized materials }\end{array}$ & $\begin{array}{l}\text { Collagen } \\
\text { Polysiloxanes } \\
\text { Monolayers } \\
\text { Cellulose }\end{array}$ & $\begin{array}{l}\text { Ca phosphate } \\
\mathrm{CaCO}_{3}, \mathrm{CaSiO}_{3} \\
\mathrm{Fe}_{2} \mathrm{O}_{3} \\
\mathrm{CaCO}_{3}\end{array}$ \\
\hline
\end{tabular}


structural supports, porous filtration media, grinding and cutting tools, lenses, gravity sensors, and magnetic guidance systems. An understanding of the physical mechanisms by which these organic molecules manipulate the structure of crystals and crystal surfaces can enable us to produce a new array of tailored, crystalline microstructures for application across a wide range of technologies.

The purpose of this proposal is to understand the interplay between solution chemistry, molecular structure, surface chemistry, and the processes of nucleation and crystal growth with the goal of forming the physical basis of a competency in solvent mediated self-assembly. The questions we will address include:

- How do systematic variations in the structure of the molecular building blocks in the crystal affect the kinetics and morphology of assembly?

- What are the minimum energy pathways by which growth units leave the solvated state to enter into the crystal?

- How do changes in the solution chemistry such as $\mathrm{pH}$, ionic strength, and supersaturation alter the kinetics and energetics of growth?

- How do organic additives such as proteins common in biomineralizing systems interact with the solvated species and the crystal surface to produce morphological and structural changes?

- How do organic templates act to alter the surface energies and activation barriers in order to control the process of nucleation?
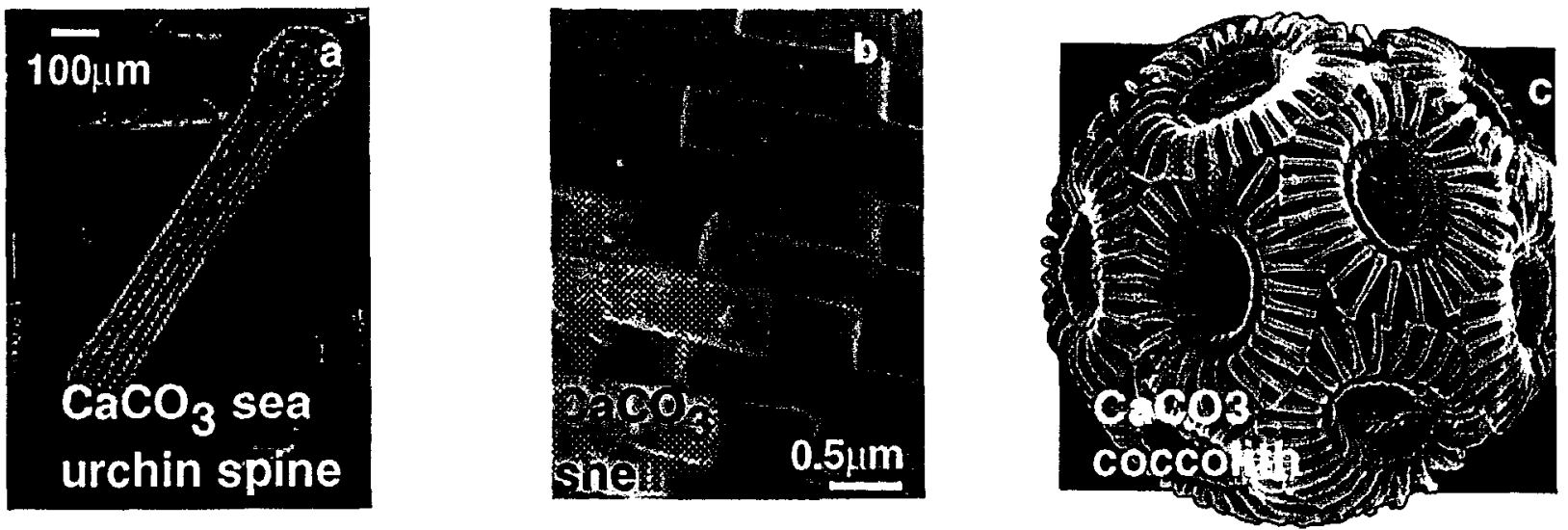

Figure 1 - Examples of crystalline structures generated through biomineralization. a.) Single crystal calcite oriented along the spine axis, b.) Abalone shell composed of a single crystal $\mathrm{CaCO}_{3} /$ polymeric-protein laminate, c.) Skeleton of a coccolithophore composed of calcite single crystals. Each element has an identical crystallographic orientation.

\section{General Approach}

Our approach to understanding the physical controls on solvent-mediated self-assembly builds upon the successful methodology developed over the past fifteen years for the study of thinfilm growth. Using scanned probe microscopy, molecular dynamics (MD), and kinetic Monte Carlo (KMC) methods, the physics of crystalline thin film epitaxy on metals and semiconductors in UHV environments has been intensely investigated both experimentally and theoretically. ${ }^{10} \mathrm{~A}$ detailed picture of growth has emerged including quantitative determinations of the relative importance of energetic and kinetic factors in controlling surface morphology.

Much less attention has been given to epitaxy in solvent mediated systems despite the fact that it forms the basis for both the organic and biomimetic approaches to nanoengineering of the solid state discussed above, as well as many other materials technologies as diverse as rational drug design 11 and corrosion science. The major reason for this lack of advancement is that, until recently, no experimental technique existed for investigating solvent-mediated systems at the nanometer scale. The advent of atomic force microscopy has changed that. In recent years, our group and others have demonstrated the capability to use atomic force microscopy (AFM) to monitor in situ growth and dissolution of single crystals of inorganic molecules, small organic 


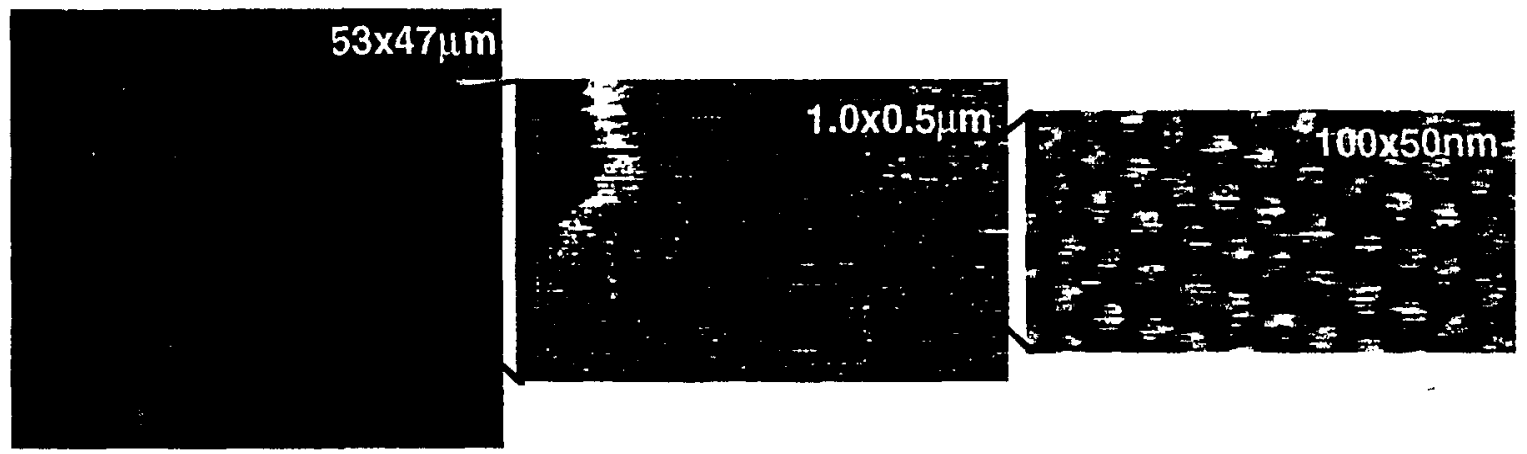

Figure 2 - AFM images of a growing canavalin crystal at different magnifications. a.) $50 \mu \mathrm{m}$ image of growth spirals, b.) $1.0 \times 0.5 \mu \mathrm{m}$ non-contact image of step-edge dynamics and oriented molecular chains on the surface, c.) $100 \times 50 \mathrm{~nm}$ image of the canavalin lattice.

molecules, proteins, and viruses with near molecular resolution in real time (See Fig 2). 12-16 The resulting AFM "movies" provide a window into the growth process that allows us to study the dynamics of evolving surface morphologies with a level of rigor rivaled only by low energy electron microscopy (LEEM) and some STM studies of metals and semiconductors, applicable only in UHV systems. While many of the questions which have been addressed for UHV systems still apply, the involvement of complex molecular or ionic species as well as the solvent layer above the solid significantly complicates the energy landscape at or near the surface.

Growth of a crystal surface from solution is a multistage process involving both energetic and kinetic factors. Figure 3 illustrates the structure of the energy landscape traversed by a solute molecule in making the transition from the solvated state to altachment at a kink site on the surface. The solute molecule starts out at high free energy in the solvated state. In order to become part of the solid it must be thermally activated through a series of energy barriers and wells associated with some combination of desolvation, adsorption, diffusion, and incorporation at a kink site. The kinetics of growth is controlled by the heights of the barriers while the equilibrium morphologies and growth modes are determined by the depths of the wells. The goal of the proposed effort is to provide a physical picture of growth which includes the hydration state of the surface, the structure of the near-surface liquid layer, the pathway by which solute molecules make the transition from solvated to solid state and the relative heights and depths of the barriers and wells, as well as the effects of molecular structure and solution chemistry on these parameters.

The presence of complex molecular and ionic species and a near-surface solvent layer presents new challenges for both the experimental and theoretical approaches. In this proposal, we address these challenges by utilizing:

- An AFM approach that provides in situ, real time imaging of growth from solutions

- $A b$ initio quantum chemistry-Poisson-Boltzmann (QC) methods to calculate the intcraction potentials of the relevant chemical species and the near-surface solution structure

- Molecular dynamics (MD) simulations based on the QC interaction potentials to identify minimum energy pathways to the solid state

- Kinetic Monte Carlo (KMC) models that include the reaction kinctics of the MD pathways

The focus is on two systems chosen to address the two length scales of assembly, the family of 2,5-diketopiperazines (X-DKPs) and the system of $\mathrm{CaCO}_{3}$ with amino acids. Using AFM, we will record the evolution of surface morphology, critical lengths, step speeds, and stepstep interactions as a function of supersaturation and temperature. In the case of the DKPs, these measurements will be repeated as the molecular structure of the growth unit is varied. In the case of $\mathrm{CaCO}_{3}$, they will be performed as a function of solution chemistry including $\mathrm{pH}$, ionic strength, and amino acid content. In addition, we will measure nucleation rates and orientations of $\mathrm{CaCO}_{3}$ on polyamino acid templates. From these measurements, we will extract fundamental growth parameters for input into KMC simulations whose predictions will in turn be compared to the 
experimental observations. The $\mathrm{KMC}$ simulations will incorporate atomic processes representing the minimum energy pathways as determined from the MD calculations. The interaction potentials of the relevant chemical species as well as the hydrated surface, including the electrochemical double layer, which go into the MD calculations will be determined using coupled solutions to the Schrödinger and Poisson-Boltzmann equations taking into account electronic relaxation effects. Figure 4 illustrates the relationships between the three modelling approaches. KMC simulates the experimental time and length scales; the MD simulations capture the $10-100$ nanometer length scale both with real time (ns) and quasistatic calculations; and the QC approach addresses the molecular length scale quasistatically.

The X-DKP and $\mathrm{CaCO}_{3}$ /amino acid
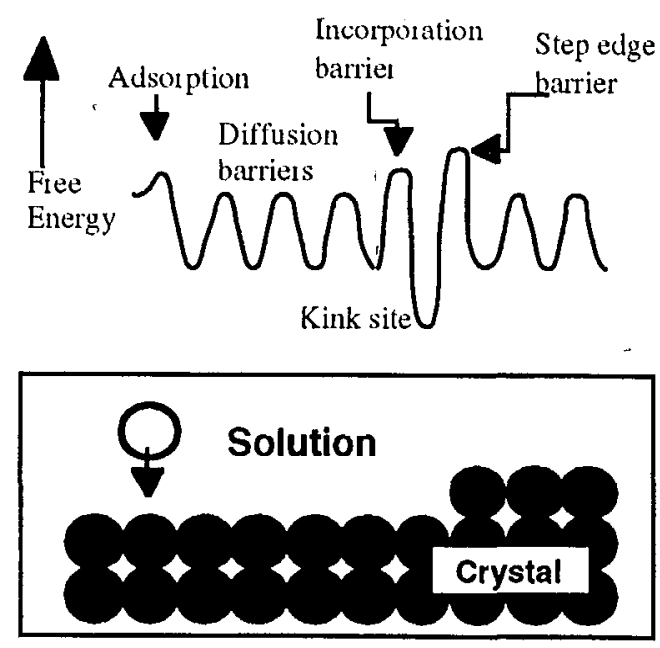

Figure 3 - Structure of the energy landscape travcrsed by a solute molecule in going from the solvated state to attachment at a kink site.

systems that will be investigated are strongly

complimentary. The experimental techniques used to analyze both systems are identical and the XDKP molecules belong to a class of cyclic dipeptides whose chemistry is closely related to that of the amino acids of interest allowing us to rcadily adapt the quantum chemistry and molecular dynamics calculations to both systems; and because the KMC models provide a general description of molecules traversing the energy landscape, they are also applicable to both systems.

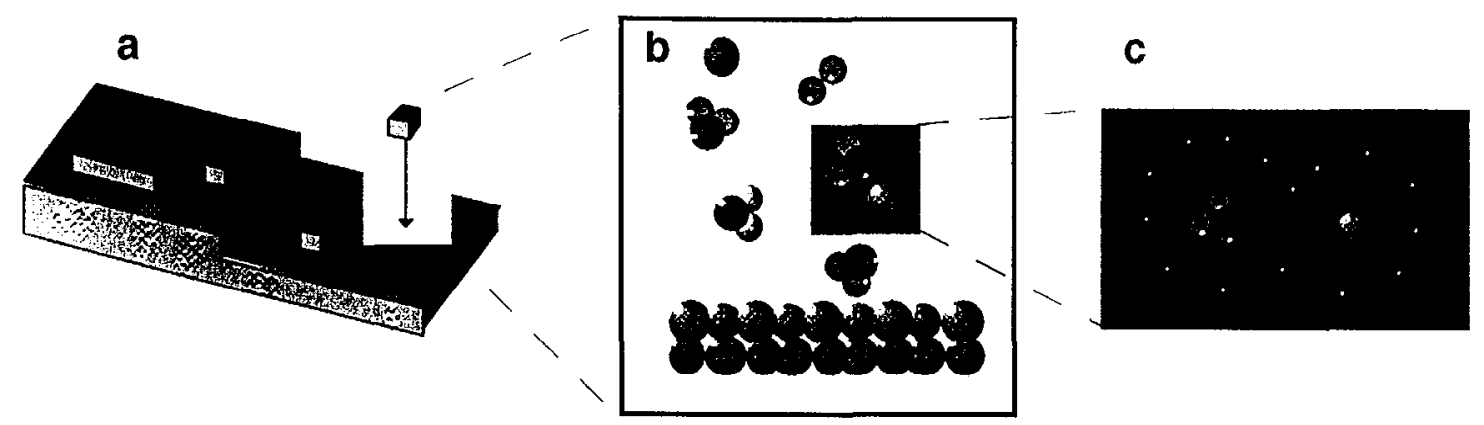

Figure 4 - Schematic illustrating the relationships between the a) $\mathrm{KMC}, \mathrm{b}$ ) $\mathrm{MD}$, and c) QC models.

\section{Background science and preliminary results} Engineering crystal structure

The overall goal of the supramolecular approach to crystal engineering is to understand the relationship between the structure of molecules and the structure of the crystals they form. Covalent interactions between atoms define the structure of the molecules but the structure of the crystals is defined by additional factors including electrostatic interactions, molecular shape, and molecular symmetry, all of which influence the arrangement of molecules within the solid. 4 Engineering the structure of a crystal requires an understanding of how and to what degree these factors influence molecular packing and how they can be used to control the assembly of molecules during crystallization and thus engineer structure at the nanometer scale.

Efforts have recently begun to exploit the directionality and selectivity of hydrogen bonds to control the orientation of the molecules in the solid.2-4,18 Judicious choice of molecular structure allows for independent control over the strength of bonding and geometry of packing in the three orthogonal directions. For example, Russel et al. ${ }^{2}$ showed that pillared two-dimensional 
hydrogen-bonded networks could be constructed in which molecular pillars separating the sheets define galleries designed to host guest molecules, with the size of the galleries defined by choice of the pillars .

The systematic approach to supramolecular engineering which has been pursued by one of us (Palmore) is to design molecules which assemble into one- and two-dimensional aggregates that serve as scaffolds on which to construct three dimensional structures. 18 Derivatives of 2,5diketopiperazines (X-DKPs) have been chosen as the molecular building blocks as shown in Fig 5a. These are particularly attractive both from the standpoint of solid-state engineering and understanding controls on growth because, while in their simplest form they generate simple onedimensional tapes, more complex molecular units and supramolecular motifs can be easily constructed by adding increasing complexity to the same basic unit as shown in Fig $5 b$. The selection of DKPs is based on several criteria that include: 1) the presence of a pair of secondary amides held in cis conformation to promote the formation of a robust, one-dimensional aggregate referred to as "tapes" (Fig 5b), 2) the near planarity and conformational rigidity of the sixmembered ring to decrease the occurrence of polymorphism caused by conformational mobility in the backbone of the tape, 3) the relative ease of synthesis, and 4) the availability of numerous avenues for systematic variation of molecular properties such as size, shape, symmetry, hydrogenbonding ability, and hydrophobicity through changes in $\mathrm{X}$, all of which can influence both the kinetics and morphology of crystallization.

Figure 6 shows a crystal of GLYDKP - a glycine derivative and simplest member of the X-DKP family - along with schematics showing the packing of GLYDKP tapes in the lattice.16 This crystal possesses a number of asymmetries. The tapes are strongly bonded along the tape

$\mathbf{a}$

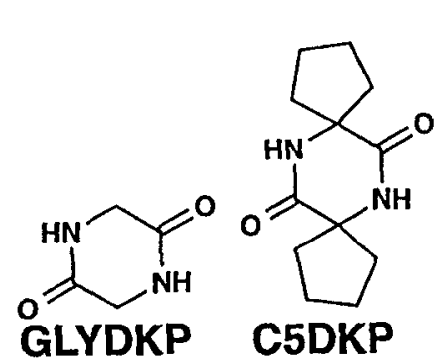<smiles>O=C1CNC(=O)[C@H](CO)N1</smiles><smiles>O=C1CNC(=O)[C@H](Cc2c[nH]c[nH+]2)N1</smiles>

b

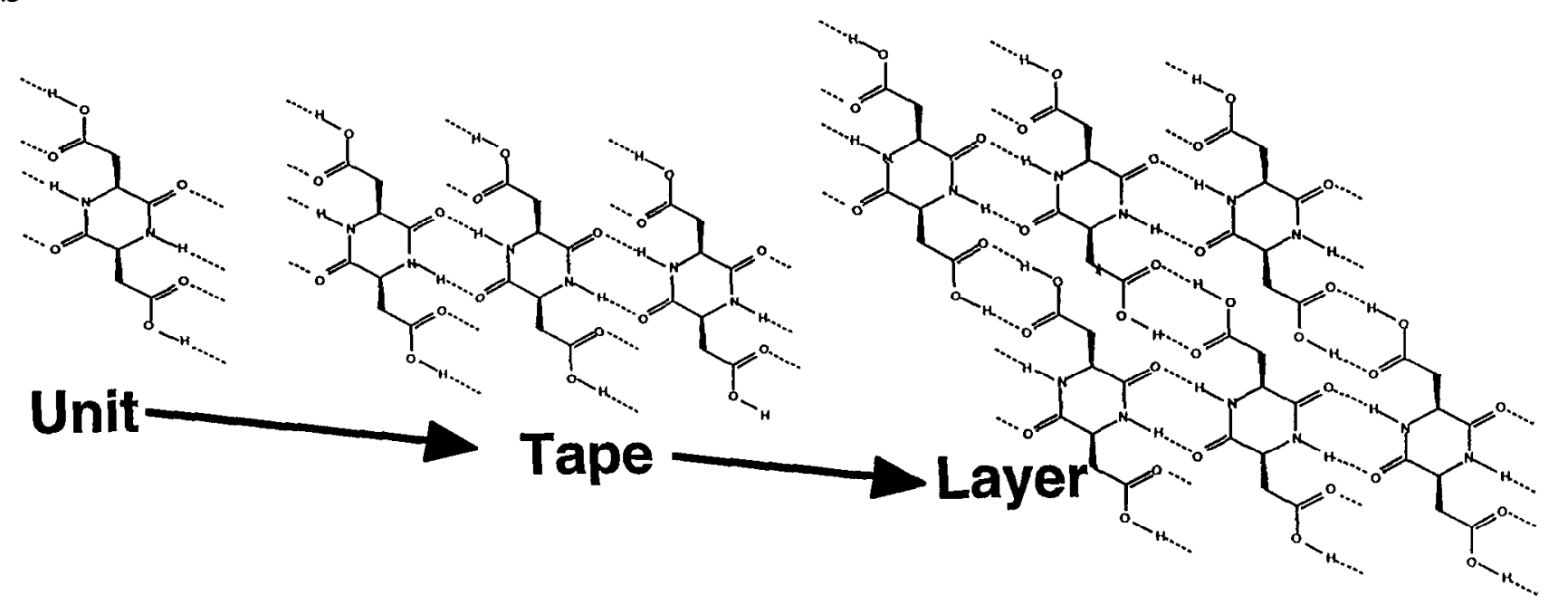

Figure 5 - a.) Structure of X-DKP molecules to be investigated. b.) Linking of individual X-DKP molecules to form tapes and layers. 
direction but weakly bonded in the two orthogonal directions; the cant angle of the tapes reverses from layer-to-layer; and the crystal possesses a $180^{\circ}$ screw axis so that the interaction between adjacent layers alternates between $\mathrm{H}-\mathrm{H}$ and $\mathrm{O}-\mathrm{O}$, the latter being more repulsive.

All of these asymmetries are reflected in the kinetics and morphology of the growing crystal at the molecular level. Figures $6 c$ and $6 \mathrm{~d}$ show AFM images of the surface of a growing GLYDKP crystal. 17 The asymmetry in bonding leads to ragged step edges along the stronglybonded tape ends and straight step edges perpendicular to the tape direction. In addition, the steps propagate an order of magnitude faster along the tape direction than perpendicular to it. The alternation in cant angle between adjacent layers leads to alternate step-doubling as, on one side of a terrace, the fast step of an upper terrace overtakes the slow step on the terrace below, while on the other side of the terrace the situation is reversed. Finally, due to the alternation between $\mathrm{O}-\mathrm{O}$ and $\mathrm{H}-\mathrm{H}$ interactions, double steps emitted at the dislocation sources show a similar alternation in doubling on opposite sides of the growth source. This behavior - known as step interlacing arises from three separate causes: differences in bond-strength, packing, and repulsion. This initial data on the simplest member of the XDKP family demonstrates that the ability to control the complexity of the shape, bonding, and packing of the molecular growth units provides a source of rich behavior and an ideal testbed for theoretical models of solvent-mediated growth.
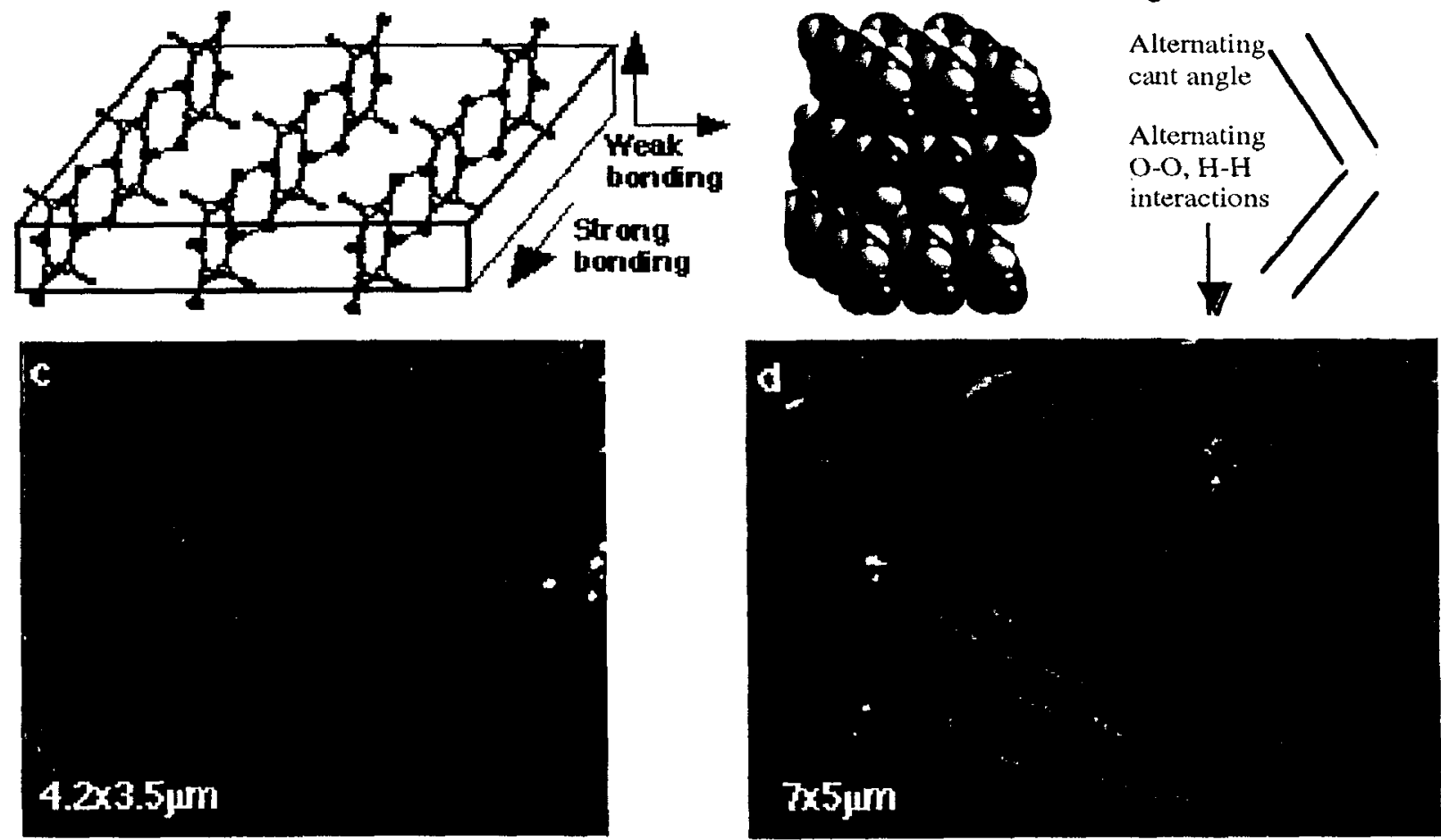

Figure 6 - a.) Schematic of GLYDKP structure and b.) asymmetry of molecular packing in crystal, c.) and d.) AFM images of GLDKP growth spirals showing step interlacing, and anisotropy in step speed and step roughness.

\section{Engineering crystal form}

In controlling crystal growth at the micron to millimeter scale, living organisms utilize a diverse array of strategies to generate biomineralized structures, including synthesis of clusters and nanoparticles, growth and assembly of single crystals and shaped composites, and microstuctural fabrication of mineral-polymer ceramic-like composites and organized materials (see Table I). As Table I shows, $\mathrm{CaCO}_{3}$ - in the form of calcite and aragonite - is ubiquitous among biomincralized structures. Recent biological and biochemical studies of biomineralization suggest that calcifying organisms have adopted strategies for controlling morphology based on the release of functional organic molecules. 19 The fascinating complexity of this control is illustrated in a comparison of the external form of single calcite crystals deposited as coccolith segments -as, for example, in Fig 1 - with a typical rhombohedral inorganic calcite crystal. 20 
The organic mixtures believed to be responsible for biologically-directed mineral growth have complex and diverse chemistries. For example, mollusks are rich in aspartate $(30 \%)$ and glutamate residues while some algae coccoliths are associated with carboxylated polysaccharides. ${ }^{21}$ This complexity may explain the surprisingly few studies that have attempted to investigate mechanisms by which organic molecules modify calcite morphology. Berman et $a l .22$ found that the habit of calcite crystals changed when grown in the presence of sea urchin proteins and Belcher et al. 8 showed that proteins from abalone shells could be used to switch the crystallizing form of $\mathrm{CaCO}_{3}$ back and forth between aragonite and calcite. Other investigations

have examined the inhibitory effects of some macromolecules in solution.9 However, the chemistries of these naturally occurring systems are too complex for isolating the molecular controls of individual molecules on growth morphology.

In a more fundamental approach, Didymus et al. 20 investigated the inhibitory effects of simple oxyanions on calcite habit. A comparison of resulting crystal morphologies by SEM showed that decreasing the chain length between the terminal carboxylates of different additives correlated with the increasing elongation of crystals with roughened and curved prismatic faces. They explained this change by a cooperative binding mechanism of carboxylate groups with calcium atoms at the mineral surface. Addadi et al. ${ }^{9}$ found that calcite formed in polyaspartic acid exhibited (001) faces while those grown in polyglutamic acids did not. These investigations offer tantalizing insights to the control of simple carboxyl groups on calcite morphology and suggests that structure directing effects are systematically related to the configuration of the functional ionized groups.

When considering the controls of organic compounds on inorganic crystallization, it is important to distinguish between the stages of nucleation and growth. The common association of organic compounds with biomineralized structures, $\mathbf{5 , 8 , 9 , 1 9 , 2 1}$ the gross morphological changes induced by the addition of these compounds to crystallizing systems $\mathbf{7 , 8 , 2 2}$ as well as their effect on crystallization kinetics,, 23 and the variations in $\mathrm{x}$-ray rocking curve widths observed among naturally occurring $\mathrm{CaCO}_{3}$ structures ${ }^{22}$ all demonstrate that these compounds modify the growth stage of minerals. In addition, there is a substantial body of evidence to suggest that proteins and other organic molecules can and do serve as nucleation "templates", providing preferential sites for nucleation and controlling both the orientation and stable growth facet of the resulting crystals. ${ }^{24-28}$ Many organic-inorganic composites including the shells of bird eggs 24 and mollusks ${ }^{25}$ exhibit a layered structure in which the inorganic component grows at the macromolecule-solution interface. A number of experimental studies $9,26-28$ have used organic films to mimic this process. However, no investigations into the physical mechanisms of this process have been performed, nor have the systematic relationships between functional groups on amino acids and the rates and mechanisms of nucleation control been explored. In fact, little effort has been made to understand the reaction mechanisms or physical basis of these processes.

We are currently pursuing an experimental investigation of $\mathrm{CaCO}_{3}$ growth with and without the presence of amino acids. ${ }^{29}$ Figure 7 a shows the morphology of a growth hillock on the $\{10 \overline{1} 4\}$ face of calcite. This face advances on anisotropic, polygonal dislocation hillocks which exhibit well defined steps along the two [ $\overline{4} 41]$ and [481] microfacets. The geometry of the growing face is shown in Fig $7 \mathrm{~b}$ along with the distribution of kink sites along the steps. ${ }^{30}$ There are four types of kink sites (labeled 1 - 4 in Fig 7b) characterized by differences in bond distributions. For example, the three coordinating oxygen atoms of the type 1 kink lie within a single carbonate layer while those of the type 4 kink site lie in two adjacent layers resulting in a more closed site. These differences result in a pair of steps that make one obtuse angle and one acute angle with the surface. We should expect this anisotropy in kink site bond distribution to be reflected in kink site activity and/or surface diffusivity and, hence, in step speed and hillock slope, resulting in two pairs of equivalent hillock sectors. And, in fact, we find that the obtuse steps 

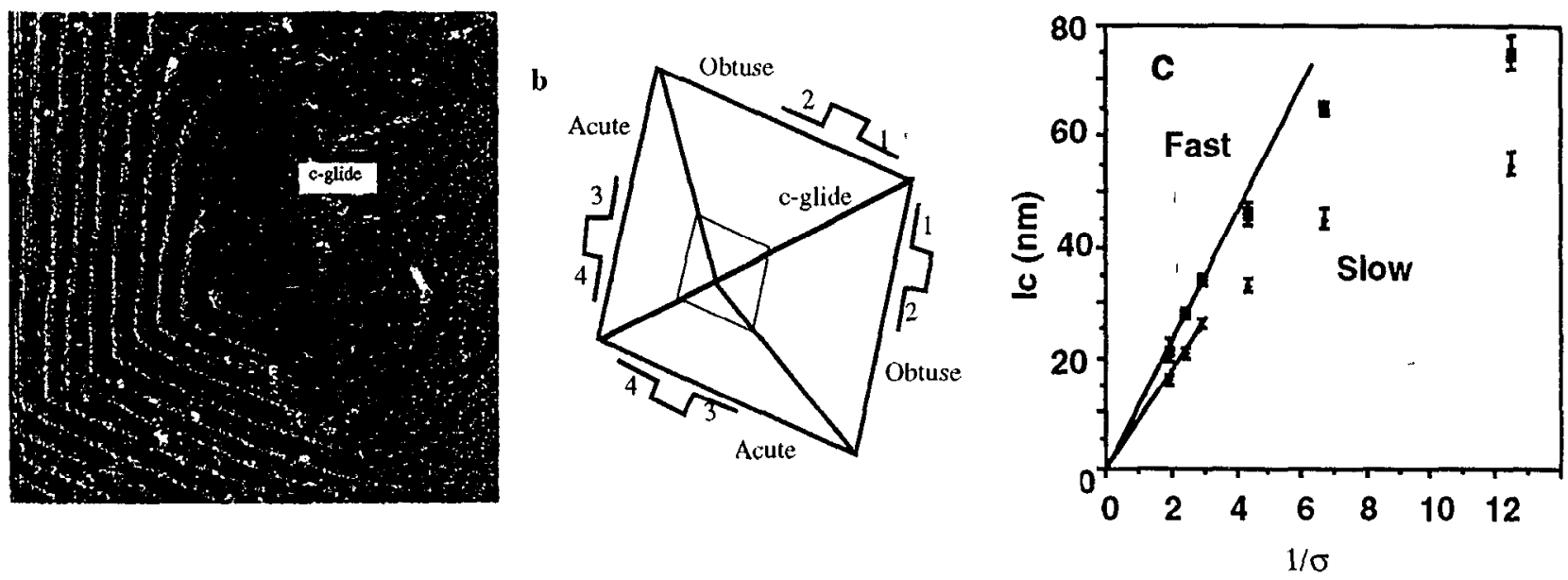

Figure 7. a.) $5 \times 5 \mu \mathrm{m}$ AFM image of a growth hillock on a calcite $\{10 \overline{1} 4\}$ face. b.) Schematic of hillock showing geometry of the four types of kink sites. c.) Dependence of critical length on supersaturation.

grow faster than the acute steps. We should also expect changes in solution chemistry to modify the step kinetics and surface morphologies of the crystal by selectively altering the activity of the kink sites. Figure 8 shows the effect of aspartic acid and glycine on the etchpit microfacet directions during dissolution. The addition of the amino acid alters the microfacet directions either through changes in the step edge energies or step kinetics. Other measurements show that changes in the ionic strength and/or $\mathrm{Ca}^{2+}$ to $\mathrm{CO}_{3}{ }^{2-}$ ratio also leads to variations in step kinetics and hillock geometry. These preliminary results illustrate the rich behavior exhibited by this system and the dramatic potential of organic additives for modifying crystal morphology at the molecular level.
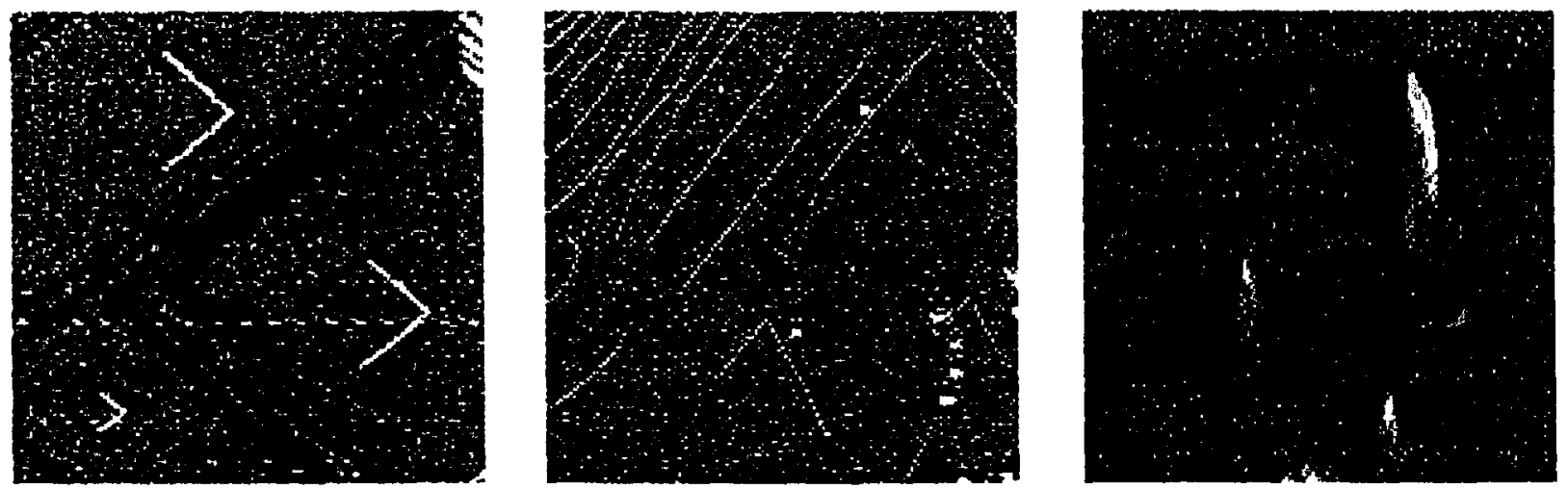

Figure 8 - AFM images of dissolution pits on the calcite $\{10 \overline{1} 4\}$ face under a.) pure conditions (water), b.) with aspartic acid, and c.) glycine.

\section{Application of crystal growth fundamentals}

As a solute molecule approaches the crystal it encounters an electrostatic potential due to the crystalline surface charge and its associated counterion distribution, the so called electrochemical double-layer. If the solute molecule attaches directly to the kink-site, an intermediate solute-kink complex may form with a higher free energy, creating a barrier to altachment over which the molecule must be thermally activated. If the pathway to the kink site is instead via surface diffusion, then the solute molecule must overcome the barrier of partial desolvation to adsorb to the surface. Its attachment to the kink site is then preceded by a series of activated hops along the surface. Because the presence of the step disturbs the surface periodicity, the barriers near the step edge will, in general, be different than those on the isolated terrace. As a consequence, the barrier to attachment may be different for approach to the step from below versus approach from above, 
producing a diffusion bias commonly referred to as the Schwoebel-Ehrlich effect. The energy landscape associated with these processes is shown schematically in Fig 3.

Consistent with this general picture of growth, a formalism for quantitatively relating the morphology and kinetics of growth to the energy landscape based on equilibrium thermodynamic considerations was developed. 31-34 It provides relationships between experimentally adjustable parameters such as temperature and supersaturation and measurable quantities such as island sizes and shapes, critical step lengths, and step speeds. Application of this formalism to AFM data allows us to extract fundamental growth parameters for input into and comparison with KMC simulations.

The need for coordination between experiments, the classical growth formalism and the $\mathrm{KMC}$ approach is illustrated by the measurements of the critical length during growth of calcite. At the low values of supersaturation, $\sigma$, typical in solvent mediated systems, growth occurs primarily on dislocation-generated growth spirals such as that shown in Fig $7 \mathrm{a}$ on a calcite $\{10 \overline{1} 4\}$ surface. Measurement of the dependence of the length of the first turn of the spiral on supersaturation, $\sigma$, gives the critical length, $\mathrm{l}_{\mathcal{C}}$, directly, and the step edge free energy, $\alpha$, through the relationship $\mathrm{l}_{\mathfrak{C}}=$ $2 \omega \alpha / k T \sigma$, where $\omega$ is the molecular volume in the solid, and $\mathrm{kT}$ is the thermal energy. 31,32 The dependence of $l_{\mathrm{C}}$ on $1 / \sigma$ as measured from sequences of images such as Fig $7 \mathrm{a}$ is given in Fig $7 \mathrm{c}$. Whilc at high supersaturation the dependence of $l_{\mathcal{C}}$ on $1 / \sigma$ is linear, extrapolates to zero, and gives the value of $\alpha$, at low supersaturation (large $l_{C}$ ) the dependence of $l_{c}$ on $1 / \sigma$ deviates from the equilibrium thermodynamic prediction, indicating that kinetic factors must be considered. These are accessible through $\mathrm{KMC}$ simulations.

The need to combine the experiments with both classical theory and a KMC approach is further emphasized by considering the kinetics of step advancement. The thermodynamic driving force for growth is the difference in free energy between the initial and final states $(\Delta \mu=k T \sigma)$, but the rate at which growth proceeds is controlled both by $\Delta \mu$ and by the largest energy barriers in the energy landscape. There is a considerable body of data (see for example, refs. 15, 35-37) as well as theoretical considerations $\mathbf{3 2 , 3 3}$ which show that in many aqueous systems, over a wide concentration range, this relationship is given by $v=\omega \beta\left(C-C_{e}\right)$ where $v$ is the step speed and $C$ and $\mathrm{C}_{e}$ are the actual and equilibrium concentrations, respectively. This relationship is expected to hold even when the systems is well mixed so that growth is not limited by bulk diffusion, provided step advancement is not limited by the availability of kink sites (rough step approximation). The kinetic coefficient, $\beta$, is generally assumed to be of the form $\beta=\beta_{0} e^{-E / k T}$ and relates the admolecule current to the steps (i.e., the step speed) to the solute concentration, $C$. At least in some systems $\beta$ does exhibit Arrhenius behavior, 37 and the dependence of $\beta$ on step-step spacing matches the predictions of a surface diffusion model.15,36 However, the parameters which go into $\beta$ must take into account the multiple steps involved in getting from the solvated state to the crystalline state. Thus the details of the dependence of $\beta$ on atomistic parameters are model specific ${ }^{32,33}$ which, while not easily addressed analytically, can be readily incorporated into KMC models, particularly when the temperature dependence of $\beta$ is known. Our measurements of the dependence of step speed on $\mathrm{C}-\mathrm{C}_{\mathrm{e}}$ for the two distinct types of steps on the calcite $\{10 \overline{1} 4\}$ surface show that, while step speed is linear in $\mathrm{C}-\mathrm{C}_{\mathrm{e}}$ for the fast step direction, the slow step exhibits a nonlinear dependence which may be explainable in terms of kink site kinetics. But the classical models do not allow us to adequately account for the myriad of potential kinetic processes. Application of atomistic KMC simulations allows the potential sources of such behavior to be identified. 
Other comparisons between classical theory and AFM experiments have given important information on activation barriers and surface energies for 2D nucleation,, 38 the diffusion length of adsorbed molecules $\mathbf{1 5}$ and the effects of stress on critical length. 37 The salient point for the purposes of the proposed effort is that by using the AFM to measure the changes in surface morphologies, hillock geometry, and step kinetics, one can determine the effects of altering the structure of the solute molecules, changing the solution chemistry or modifying the surface chemistry on the step-edge free energies, activation barriers, molecule-step and step-step interactions, diffusion lengths, and surface energies. Most importantly, interpreting the data in terms of atomic processes requires an atomistic modeling effort.

\section{Modeling Solvent-mediated growth}

Our understanding of the physical and chemical basis of solvent-mediated self assembly of solids will be enhanced by a strong computer modeling effort rooted in fundamental theoretical principles. Our methodology must provide a path for meso-scale calculations which can be compared with experiment. To this end, we require a hierarchy of computational techniques which enable us to incorporate information obtained on one length scale into calculations that address another length scale.

Monte Carlo Models: Kinetic Monte Carlo (KMC) simulations provide a direct link between experimentally observed morphology and atomistic processes. It has long been the method of choice for modeling the long time evolution of crystal growth and dissolution. Monte Carlo methods have been applied to the study of island growth during metal-on-metal epitaxy,39 growth of compound semiconductors $\mathbf{4 0 , 4 1}$ surface roughening and scaling, 42 the growth of spirals from solution, 43 and the dissolution of calcite. 44

The application of KMC to the study of crystal growth relies on the simple picture of atoms (or growth units) evolving along the energy landscape shown in Fig 3. The rate at which an atom can move from one minimum to the next is assumed to have the Arrhenius form

$\left(\mathrm{h}=\mathrm{ve}^{-} \mathrm{E}_{\mathrm{b}} / \mathrm{kT}\right.$ ) where $\mathrm{v}$ is proportional to the attempt frequency of the event and $\mathrm{E}_{\mathrm{b}}$ is the height of the barrier between the initial and final state. At any given step in the simulation, the probability that a particular process may occur is proportional to its rate. A process is selected and carried out (e.g., movement of an atom along the surface), the time step for the event is computed stochastically, $\mathbf{4 5}$ and the rates for additional movement are recomputed in response to the event. Hence, KMC provides a statistical method for the real time evolution of a surface through the motion of the individual growth units.

In order to apply KMC methods, the position of the energetic minimum, the transition rates, and the processes by which the system may evolve from one minima to the next must be known a priori. The accuracy of the method is only as good as the processes and rates included into the simulation. This information must come from experiment and/or more detailed quantum mechanical or molecular dynamics simulations.

Molecular dynamics: Metropolis Monte Carlo (MC) and molecular dynamics (MD) calculations have been used to study the structure 46,47 and dynamical processes 48 at crystalsolvent interfaces. This region has long-range (in plane) atomic structure and high ionic concentrations making continuum approximations invalid, but it can be treated using MD/MC methods. However, due to long range electrostatics and chemical equilibrium considerations, the effects of the solvent-solute solution far from the surface needs to be included. Continuum methods for $\mathrm{pH}$ and concentrations of interest are well suited to modeling the bulk and the "transition to bulk" (diffuse layer) regions. The bulk solution region controls the chemical potentials whereas the diffuse layer controls the electrostatic potential at the interface.

Under the assumption that the bulk region and diffuse region become uncorrelated from the surface region except for charge neutrality, the effects of the bulk and diffuse regions can be replaced with a set of chemical potentials to model the particle source/sink of the bulk, a fixed sheet of charge to model the diffuse layer, and a soft repulsive interaction to model the remaining short range atomic interactions. The chemical potential can be calculated for a given bulk solution 
via a chemical equilibrium code using Debye-Huckel theory. The charge on the sheet is fixed by overall charge neutrality. For dynamical properties a tractable approach is to employ a constant pressure, temperature, and particle number MD method with pressure and particle number fixed using the above grand canonical MC method. The MC/MD approach requires a set of potentials to describe the interaction between solvent and solute, solvent and surface, and solute and surface.

For many of the interactions of interest, empirical potentials have been developed. 49 Quantum chemical calculation will be used to both test and modify these potentials as well as to develop new potentials as needed.

Quantum chemistry: We have developed a method for incorporating solvent effects, including ionic strength, into $a b$ initio electronic structure determinations, 50,51 a technique which is directly applicable to solvent-mediated assembly. Briefly, a molecular or solvent-accessible surface surrounds the molecule, providing an interface between the internal region of the molecule and the solvent. An $a b$ initio quantum calculation can then be performed at the $6-31 \mathrm{G}^{*} * / \mathrm{MP} 2$ level of theory which provides the electrostatic fields to be used as the source terms at these surface elements in a Green's function-based Poisson-Boltzmann calculation. This calculation then provides the single-and double layers of induced polarization charge at the molecular surface.

In the next iteration, the quantum calculation is repeated, this time with the induced surface charges from the Poisson-Boltzmann (PB) calculation included in the Hamiltonian. The result is a new set of electric fields at the surface elements which again provide the sources for the PB calculation. Again, the induced surface charges are added to the Hamiltonian. The calculation is iterated until convergence is achieved.

In this way, the electron distribution relaxes in response to the single and double layers of charge, a significantly different approach from classical methods in which the charge distribution is fixed. Whereas these classical methods utilize fixed partial charges obtained. e. g., from vacuumstate quantum calculations, in our method, partial charges can be obtained when required from the fully relaxed electronic charge distribution in the presence of solvent. This method has been applied to the calculation of the free energy of solvation of the carbonate ion, $\mathrm{CO}_{3}{ }^{2-}$, and found to be in excellent agreement with experiment. 50

From this procedure we find the potential and electric field at the surface determined by the $a b$ initio calculation in the presence of both single and double layers of charge. We have produced an "object," a Molecular Single and Double Layer (MSDL) containing the relaxed electronic charge distribution and the single and double layers of charge. This MSDL can then be used to determine the potential and electric field outside the molecule, e. g., at the surface elements of another such MSDL. The electrostatic interaction between the molecules is dependent on molecular orientation, the dielectric constant, ionic strength, and $\mathrm{pH}$ of the solution. Each molecular type (amino acid; 2,5-diketopiperazine) is treated individually in its proper $\mathrm{pH}$-dependent protonation or charge state.

The electrostatic interaction between a large number of these MSDLs is obtained by summing over these single and double layers of surface charge. The problem is amenable to fast multipole and Greengard-Rocklin algorithms which greatly speed the process of minimization of the total energy of a system of these MSDLs. The MD calculations then provide the path from these fundamental quantum determinations of interactions to an assembled" structure through KMC models.

\section{Proposed work}

\section{Experimental approach}

Our basic experimental strategy is to use AFM to measure the dependencies of step speeds, critical lengths, nucleation rates, terrace widths, and step-step interactions on molecular structure and solution/solute composition. Imaging is performed in a fluid cell where the solution is kept flowing at a rate sufficient to maintain a constant saturation state within the cell. Currently, the temperature inside the fluid cell can be varied over the range of $15-40^{\circ} \mathrm{C}$ and controlled to within $0.5^{\circ} \mathrm{C}$. For the proposed work, this range will be extended to $10-90^{\circ} \mathrm{C}$ through minor 
modifications to our experimental setup. Additionally, the solutions can be changed "on the fly" without interrupting the imaging. This allows direct comparison of effects of solution variations.

Individual images provide direct information about step directions and roughness, anisotropies, general morphology, and growth mode (step flow vs. island nucleation). When viewed sequentially, they form movies of the evolving surface morphology. From these movies, the step velocities, nucleation rates and critical step lengths are measured.

In addition to AFM imaging, single crystal X-ray diffractometry will be used to determine crystal structures.

Molecular structure: During the first six months, we will perform measurements of the step velocities and critical lengths as a function of supersaturation and temperature for GLYDKP, the simplest member of the X-DKP family. Given the importance of step-step interactions, we will also determine the dependence of step speed on step spacing and attempt to investigate the dependence of $2 \mathrm{D}$ nucleation on terrace width at high supersaturation.

Following completion of the measurements on GLYDKP, we will systematically collect data on each of the other members of the X-DKP family starting with ASPDKP. The presence of the aspartic acid group leads to cross linking of the tapes to form connected nets. The interlayer bonding, however, remains weak. In addition to providing a strong variation in molecular structure and bonding, the functional groups of this molecule bear a strong resemblance to the amino acid additives to be used with $\mathrm{CaCO}_{3}$ and make an immediate connection through the QC and MD modeling. We expect that each system will require about six months to fully explore so that work will extend well into the third year of the project.

Solution chemistry: The pure calcite system offers a richness of parameters with which to explore the systematics of solution induced changes in crystal morphology. Our preliminary results are providing a reconnaissance survey of this system over a very limited range of solution chemistries including fixed $\mathrm{pH}$, ionic strength, and aspartic and glutamic acid concentrations. But the variations in morphology and kinetics induced by changes in these factors will provide the best testbed for our theoretical modeling effort to understand the atomistic processes controlling assembly. We will vary the supersaturation from 0.5 to 10 , the ratio of $\left\{\mathrm{Ca}^{2+}\right\}$ to $\left\{\mathrm{CO}_{3}{ }^{2-}\right\}$ from $0.1-10000$, ionic strength from 0.005 to $0.5 \mathrm{M}$, the $\mathrm{pH}$ from 7.8 to 9.0 , and the temperature from 10 to $90^{\circ} \mathrm{C}$. The supersaturation determines the driving force for crystallization and by changing it, we change the degree to which the system is out of equilibrium. Varying the ratio of $\mathrm{Ca}$ to $\mathrm{CO}_{3}$ provides a way to probe the competition between calcium and carbonate species; the $\mathrm{pH}$ determines the ratios of carbonate species (predominately $\mathrm{HCO}_{3}^{-}$and $\mathrm{CO}_{3}{ }^{2-}$ ) as well as the surface charge; the ionic strength controls the shielding between charged species; and the temperature determines the degree of molecular mobility. While the general effect of amino acids on morphology and kinetics will be observed during our preliminary work, the first nine months will be primarily devoted to establishing this database for the pure system.

The addition of amino acids to the growth solution provides a way to probe the interaction between organic and inorganic materials. Aspartic acid and glutamic acid have a carboxyl side group which is thought to chelate calcium. Glycine is another amino acid very commonly found in biomineralizing proteins associated with shell formation. While it is not anticipated that the individual amino acids will reproduce the effects of the much more complex proteins, the systematic evaluation of the effects of the components is more likely to reveal the nature of organic controls and provides a more tractable problem for the modeling effort. 52 Based on the results of the first phase of research, we will choose solution conditions that represent limits of morphological and kinetic behavior as starting points for a systematic investigation of amino acidcalcite interactions. During the second phase of the project, we will introduce aspartic acid, glutamic acid, and glycine into the system to learn how the individual amino acids bind to the surface and affect calcite growth morphology and kinetics. Finally, we will determine the effects of peptide chains of poly-aspartic and poly-glutamic acid to look for cooperative effects of regularly spaced functional groups.

Organic templates: In order to investigate the control of amino acid functional groups on nucleation rates and crystal orientation during calcite nucleation, we will use poly-aspartic films 
as a template for nucleating calcium carbonate crystals. Our approach will mimic that of Berman et al. 21 and Addadi et al. ${ }^{9}$ in which poly-aspartic acid in the $\beta$-pleated sheet conformation is adsorbed onto sulfonated polystyrene substrates. By measuring the dependence of nucleation rate and critical island size on supersaturation, we can determine the activation energy for nucleation and the surface free energy. Initial imaging of this process will be performed during the first nine months, but complete investigation of the kinetics and energetics will occur during the third year of the project.

\section{Theoretical approach}

Experimental measurements will be compared directly to the results of kinetic Monte Carlo simulations and analytic models of the growth of calcite and organic solids. Initially, simple rules will be assumed for the energetic barriers and reaction pathways in order to test the effect of atomistic processes on crystalline morphology (e.g., predominant step orientations, step velocity, and roughness, critical length for growth of a spiral) and provide limits to the various rates dictated by consistency with experimental observation. The rate limiting processes are currently unknown for the systems of interest. The KMC simulations provide a testing ground for possibilities that will be examined more closely with atomistic simulations and electronic structure investigations. In turn, the KMC simulations test the effect of the processes derived from the fundamental models of the crystal solution interface.

Because much of the underlying physics and chemistry is common to assembly of both the calcite and X-DKP systems, we restrict our discussion to one system, but it has immediate relevance to the other. The calcite surface has two types of straight step edges (described by either an obtuse or acute bond angle with the layer below) and four types of kinks. In studies of dissolution, 44 this has been modeled as a cubic system consisting of only one species, three kink energies (two of the four kink types are expected to be close in energy), and a kink pair formation energy (removal of a single unit from the step edge). Reasonable agreement was obtained between simulations and experiment for the dependence of step velocity on the size of an etch pit while reproducing the extremely straight steps observed in AFM images. 44 A similar model will be applied to set a bound on the relative rates of double kink nucleation and the growth rate of kinks. Unlike dissolution, however, transport of material to the step can be the rate limiting process. Ions can attach directly to the kink sites from solution or by diffusing to the kink sites along the surface. The relative importance of direct attachment and surface diffusion is currently unknown. However, the dependence of step velocity on step separation and supersaturation will give insight into which path dominates the kinetics.

Simulated surfaces will be constructed which accurately represent the structure of the crystal surface and the large anisotropies of bonding that occur for these molecular solids. The following set of assumptions will be made for the early simulations:

(1) The concentration of ionic species in the solution above the surface is uniform (well stirred).

(2) The adsorption rate has an Arrhenius form with a prefactor proportional to the concentration of the species in solution. The activation barrier for attachment is expected to depend on the structure of the surface.

(3) Energetic barriers for ions (molecules) to detach from the surface or diffuse along the surface are proportional to the number of nearest neighbors, the type of nearest neighbor, and the direction of the bonds (accounting for anisotropies).

The equilibrium concentration of species at the surface results from the balance of attachment and detachment. This set of assumptions is consistent with growth being surface reaction limited. The barrier to attachment may be caused by the necessity to break a bond or by the presence of an ordered liquid layer at the surface. Quantum-chemical calculations coupled to solutions of the Poisson-Boltzmann equation (QM-PB) will provide insight into the details of this barrier.

The atomic studies will first consider equilibrium structure of the crystal surface and, in particular, how this structure changes with changes in the bulk solution and/or solute structure. The influence of the bulk solution on the surface will be included via chemical potentials found using a multi-component chemical equilibrium code (CHEQ) and Debye-Huckel theory. With these chemical potentials, grand canonical MC methods will be used to find the structure of 
surfaces under solution. The effects of ionic concentration and $\mathrm{pH}$ will be determined for both flat and stepped surfaces.

These equilibrium structures will be used as a starting point to understand the dynamics. Using the Quantum mechanical-Poisson-Boltzmann approach, critical positions in the energy landscape will be explored to find adsorption sites and likely diffusion paths along the surface. To directly investigate the dynamical process, constant pressure, temperature, and particle number MD will be used to explore adsorption/desorption process, diffusion along the surface as well as step edges, and the role of collaborative effects in these process.

One of the differentiating strengths of the proposed work is the ability to calculate intermolecular potentials and interactions with charged and polarizable surfaces from first principles. It is well known that results derived from molecular dynamics calculations are only as good as the potentials on which they are based. "Small" molecules (up to $\sim 50$ atoms) are amenable to $a b$ initio techniques; we have developed the capability of using these techniques for molecules in the presence of a solvent electrolyte, including ionic strength effects (salt), and interacting with charged, polarizable surfaces. These fundamental interactions will be the starting point for our molecular dynamics calculations.

The effect of organic molecules on the solvent-mediated growth morphology and kinetics of step migration and pinning of an inorganic solid is expected to involve a covalent interaction. The incoming forcign molecule may form bonds with $\mathrm{Ca}^{2+}$ and $\mathrm{CO}_{3}{ }^{2-}$ constituents at critical sites along the step. Furthermore, the organic molecule does not impinge upon a perfect $\mathrm{CaCO}_{3}$ lattice, but upon a structure which has been electrostatically charged by exposure to an aqueous environment. We do not know on an atomic scale how the surface charge develops on a carbonate, but recognize, e. g., that protons in water may readily interact with $\mathrm{CO}_{3}{ }^{2-}$ surface ions and that $\mathrm{Ca}^{2+}$ ions will readily interact with $\mathrm{OH}^{-}$to form covalent bonds. The evolution of surface charge and the adsorption of organics require an extension of the methods described above. We will follow the method which was applied to the adsorption of a $\mathrm{Cl}^{-}$ion on $\mathrm{Al}_{2} \mathrm{O}_{3}$ (alumina) in an electrolyte. $\mathbf{5 1}$ There it was found that these polarization effects can reduce the binding energy of the $\mathrm{Cl}^{-}$and that a charged defect can displace the minimum and well depth of an adsorbing $\mathrm{Cl}^{-}$.

A crystal lattice will be generated and an atomic surface (thousands of atoms) constructed. An incoming molecule will interact chemically with neighboring ions in the lattice; that is, there is a redistribution of electrons in the neighboring environment which can only be understood quantum mechanically. Beyond this region, polarization and atomic rearrangement effects may influence the behavior of the incoming molecule, effects which may be treated by atomic and continuum methods. Here, we will surround the molecule and its immediate neighboring $\mathrm{Ca}^{2+}$ and $\mathrm{CO}_{3}{ }^{2-}$ constituents with a virtual molecular surface, a two dimensional interface which encapsulates the quantum region, separating it from a polarizable dielectric continuum. The surface will be discretized into boundary elements as described above, but the elements of that surface will appropriately interface with the dielectric constant of the lattice or to the solvent.

The electric field due to the carbonate lattice - including surface defects such as vacancies and charged adsorbates - produces a polarization - an induced charge - at the surface elements of the enclosed quantum region. These charges will be included with those produced by the internal electronic distribution in the iterative Schrödinger-Poisson-Boltzmann calculation described above. This method will provide the energy of an adatom on a carbonate surface as a function of its distance from or along the surface, and will be used as an aid in understanding the surface charge evolution of carbonates and the adsorption of organics at steps in these materials.

\section{Facilities}

De Yoreo's laboratory has two atomic force microscopes capable of operating in contact, tapping or lateral force mode. One is equipped with a flow system that allows continuous exchange of growth solution, continuous filtration of that solution, use of an air trap, and "on-thefly" exchange of growth solution reservoirs. The lab also has wet chemical capabilities, $\mathrm{pH}$ and $\mathrm{Ca}^{2+}$ probes for monitoring and controlling solution composition, ovens, hoods, and a work 
station for chemical equilibria calculations, analysis, and image processing. In addition, the Department of Chemistry at U.C. Davis has two atomic force microscopes operating both in contact or tapping mode with an optical microscopy stage and electrochemical capabilities. Prof. Palmore's laboratory is equipped with general wet chemical capabilities, a dry box, a UV-Vis-NIR spectrometer, PAR 373A Potentiostat, Solartron 1255 Impedance Analyzer and workstations for analysis and visualization of solid state structures. In addition, the Department of Chemistry is equipped with a Siemens P41RA $(\mathrm{Cu} \mathrm{K} \alpha)$ rotating anode, a Siemens P21 (Cu K $\alpha)$, a Nicolet R$3 \mathrm{~m}(\mathrm{Mo} \mathrm{K} \alpha)$, and a Scintag XDS $2000 \mathrm{X}$-ray diffractometry equipment. State of the art computing capabilities are available both at Livermore and at Sandia for performance of the proposed computational effort.

\section{Schedule of Technical Milestones}

First Year

- Modify KMC model to include crystal anisotropy, multicomponent systems, and position dependent surface flux rules

- Complete AFM data collection on pure $\mathrm{CaCO}_{3}$ as a function of supersaturation, temperature, $\mathrm{pH}$, ionic strength, and $\mathrm{Ca}: \mathrm{CO}_{3}$ ratio

- Model the effect of corner site kinetics on critical length and the dependence of step speed on control parameters using KMC code

- Combine chemical equilibria code and Debye-Huckel theory to model bulk $\mathrm{CaCO}_{3}$ solution

- Complete AFM data collection on GLYDKP and ASPDKP as a function of supersaturation and temperature

- Complete analysis of the data on morphology and kinetics for GLYDKP and pure CaCO 3

- Use QM-PB solutions to obtain interactions for $\mathrm{CaCO}_{3}$ solution species and surface

- Begin measurements of $\mathrm{CaCO}_{3}$ nucleation on poly-aspartate templates

Second Year

- Input potentials from QM-PB and Debye-Huckel into MD simulations to define minimum energy pathways for $\mathrm{Ca}^{2+}$ and $\mathrm{CO}_{3}{ }^{2-}$ ions

- Complete AFM on $\mathrm{CaCO}_{3}$ nucleation on poly-aspartate templates as a function of supersaturation and temperature

- Incorporate minimum energy pathways into $\mathrm{KMC}$ model for $\mathrm{CaCO}_{3}$

- Use QM-PB solutions to obtain interactions for aspartic acid, GLYDKP and ASPDKP

- Complete AFM on calcite/aspartic acid and GLYHIS as a function of supersaturation, temperature, and solution chemistry, start on GLYSER

- Complete analysis of the data on growth morphology and kinetics for $\mathrm{CaCO}_{3} /$ aspartic acid, ASPDKP and GLYHIS

- Apply initial KMC code to GLYDKP and ASPDKP systems

Third Year

- Modify KMC code to include aspartic and glutamic acid impurity molecules

- Apply KMC code to growth of GLYHIS and GLYSER

- Complete AFM data collection on $\mathrm{CaCO}_{3}$ /glutamic acid, C5DKP and GLYSER

- Input interactions from QM-PB and Debye-Huckel into MD simulations to define minimum energy pathways for GLYDKP, ASPDKP and GLYHIS molecules

- Incorporate minimum energy pathways into KMC model for X-DKP family

- Complete analysis of the data on growth morphology and kinetics for $\mathrm{CaCO}_{3} / g l u t a m i c$ acid, C5DKP and GLYSER

- Input interactions from QM-PB and Debye-Huckel into MD simulations to model behavior of aspartic acid and glutamic acid at $\mathrm{CaCO}_{3}$ surfaces

- Apply $\mathrm{KMC}$ code to growth of $\mathrm{CaCO}_{3}$ with aspartic and glutamic acid 


\section{Personnel and skills}

The proposed effort is a multidisciplinary collaboration between LLNL, Sandia, and the University of California at Davis. It complements an existing OBES project funded through the

Division of Geological Sciences and Engineering which is a collaboration between Prof. Patricia Dove of the Department of Earth and Atmospheric Sciences at the Georgia Institute of Technology and Jim De Yoreo at LLNL.

Lawrence Livermore National Laboratory:

Jim De Yoreo, Chemistry and Materials Science: Solvent-mediated crystal growth, AFM, crystal characterization, project management.

G. Steven Bales, Physics: Theoretical surface science, Kinetic Monte Carlo modeling, rate equation theory.

Jim Glosli, Physics: Molecular interactions, electric-double layer calculations, atomistic simulations including Monte Carlo and molecular dynamics simulations.

Chris Orme, Post-doctoral Research Associate, Chemistry and Materials Science: Experimental surface science, scanned probe microscopy, kinetic Monte Carlo modeling, protein folding, $\mathrm{CaCO}_{3}$ chemistry.

New Postdoctoral Research Associate, Physics: Quantum chemistry, molecular dynamics simulations

U.C. Davis:

Prof. Tayhas Palmore: Organic chemistry, synthesis and X-ray characterization of organic crystals.

Mary McBride:, graduate student: Organic chemistry, synthesis and X-ray characterization of organic crystals, AFM.

Sandia National Laboratory, Livermore

W.D.Wilson, Physics: Quantum chemistry, electrochemical double layer, structure of hydrated species.

\section{Resources}

\begin{tabular}{|c|c|c|c|c|}
\hline \multicolumn{5}{|c|}{ Manpower ana operaling expenses } \\
\hline & Effort (FTE) & Cost $(\mathrm{k} \$)$ & Cost $(\mathrm{k} \$)$ & Cost $(\mathrm{k} \$)$ \\
\hline J. De Yoreo & 0.5 & 122 & 127 & 132 \\
\hline S. Bales & 0.2 & 45 & 47 & 49 \\
\hline J. Glosli & 0.2 & 45 & 47 & 49 \\
\hline C. Orme & 1.0 & 110 & 114 & 119 \\
\hline W.D. Wilson & 0.5 & 122 & 127 & 132 \\
\hline Post-doc & 1.0 & 110 & 114 & 119 \\
\hline M. McBride & 1.0 & 30 & 31 & 33 \\
\hline T. Palmore & 0.25 & 30 & 31 & 33 \\
\hline Ancillary costs & - & 69 & 72 & 75 \\
\hline Total & 4.65 & 690 & $\overline{717}$ & .748 \\
\hline
\end{tabular}

Capital Equipment

1.) Computer workstation (Est. cost: $\$ 25 \mathrm{~K}$ )

Justification: The theoretical portion of this effort is heavy on computing. The Post-doctoral Associate who will work on the QC and MD simulations will need dedicated computing capability.

\section{Vitae}

\section{Jim De Yoreo}

Jim De Yoreo received his B.A. in Physics from Colby College in 1978 (Valedictorian, $\Phi B K$ ) and his Ph.D. in Experimental Physics from Cornell University in 1985. His thesis research on low temperature vibrational spectra of orientationally disordered crystals was directed by Prof. R.O. Pohl. He spent two years as a post-doctoral research associate at the University of Maine with Prof. Edward Decker modeling the thermal evolution of tectono-magmatic events and two years as a Postdoctoral 
research associate at Princeton University with Prof. Alexandra Navrotsky measuring thermophysical properties of diopside rich melts and modeling the transfer of heat by magmatic bodies. He has been a member of the technical staff at LLNL since 1989. He is currently the Section Leader for Crystal Development in the Inertial Confinement Fusion Program (ICF). He is a P.I. or Co-P.I. on OBES and LLNL Laboratory Directed Research and Development (LDRD) projects in biomineralization, an LLNL Materials Institute Project on Molecular Scaffolds, and two LDRD projects on UV induced damage in KDP crystals and development of near field spectroscopy with application to ICF optical materials. He is an elected member of the American Association for Crystal Growth (AACG) West Executive Committee, has been an organizer of 1996 American Conference on Crystal Growth (ACCG), the 1995 and 1997 ACCG-West, and is currently an organizer for the 1998 International Conference on Crystal Growth and the 1998 ACCG-West. He was co-vice chair of the 1997 Gordon Conference on Thin Film and Crystal Growth Mechanisms and will be co-chair of the 1999 conference. He has presented numerous invited talks in the field of crystal growth and has published over fifty papers on a wide range of subjects including the low-temperature vibrational spectra of glasses, the evolution of low-pressure metamorphic belts, sources of optical distortion and damage in crystals, the growth of meter-scale single crystals, and the evolution of crystal surfaces in solutions. He is a member of the MRS, the ACS, and the AACG.

\section{Selected publications:}

De Vries, S.A., Goedtkindt, P., Bennett, S.L., Huisman, W.J., Zwanenburg, M.J., Smilges, D.M., De Yoreo, J.J., van Enckevort, W.J.P., Bennema, P. and Vlieg, E., "Surface structure of $\mathrm{KH}_{2} \mathrm{PO}_{4}$ crystals growing from aqueous solution", Phys. Rev. Lett. (In review).

Land, T.A., De Yoreo, J.J., Lee, J.D., Malkin, A.J., Kutznesov, Yu. G. and McPherson, A., "In situ AFM investigation of canavalin crystallization kinetics", Surf. Sci. 384, 136 (1997).

De Yoreo, J.J., Land, and Lee, J.D. "Limits on surface vicinality and growth rate due to hollow dislocation coreson KDP \{101\}", Phys. Rev. Lett. 78, 4462 (1997).

Land, T.A., Malkin, A.J., Kutznesov, Yu. G., De Yoreo, J.J. and McPherson, A., "Mechanisms of protein crystal growth: An atomic force microscopy study of Canavalin crystallization", Phys. Rev. Lett. 75, 2774 (1995).

Malkin, A.J., Land, T.A., Kutznesov, Yu. G., McPherson, A. and De Yoreo, J.J., "Investigations of virus crystal growth by in situ atomic force microscopy", Phys. Rev. Lett. 75, 2778 (1995).

Malkin, A.J., Kutznesov, Yu. G., Land, T.A., De Yoreo, J.J., and McPherson, A. "In situ atomic force microscopy investigation of macromolecular crystallization ", Nature Structural Biology, 2, 956 (1995).

Land, T.A., De Yoreo, J.J., Lee, J.D. and Ferguson, J.R., "Growth morphology of vicinal hillocks on the \{101\} face of KDP: Evidence for surface diffusion", in: B.G. Demczyk et al., ed., Evolution of thin film and surface morphology, Mat. Res. Soc. Symp. Proc. 355, 45 (1995).

\section{Tayhas Palmore}

G. Tayhas R. Palmore received her B.S. in Biochemistry and M.S. in Chemistry from San Francisco State University in 1985. Upon graduation, she joined the research staff at Applied Biosystems Inc., where she developed new chemical methodology for the solid-phase synthesis and sequencing of peptides and oligomers of nucleic acids. She pursued subsequently a Ph.D. in Chemistry at the Massachusetts Institute of Technology, which she received in 1992. Her Ph.D. thesis on the "Synthesis and Characterization of Redox Active Polymers at Surfaces" was supervised by Professor Mark S. Wrighton. Upon graduation from M.I.T., she worked with Professor George M. Whitesides at Harvard University in 1992-1994 as an NSF Postdoctoral Fellow, conducting research in the area of fuel cells. In 1994, she joined the Department of Chemistry at the University of California, Davis as an Assistant Professor where she received a New Faculty Award from the University of California for three consecutive years (1994-1996). In 1997, she was nominated for a Camille and Henry Dreyfus New Faculty Award and received a CAREER Award from the National Science Foundation. Her recent work in the area of non-covalent interactions between molecules in solution, at surfaces and in solids has resulted in ten publications and over twenty invited presentations at conferences, workshops, and seminar series. Research done in collaboration with De Yoreo's laboratory appeared on the cover of the November issue of the International Union of Crystallography newsletter. Recently, she co-chaired a session on hydrogen bonding at the 1997 Gordon Research Conference on Physical Organic Chemistry. She leads a research group of eleven students and currently receives funding from the National Science Foundation, the Office of Naval Research, the Petroleum Research 
Fund, the California Space Institute, and the Materials Research Institute at Lawrence Livermore National Laboratory. She is a member of the American Chemical Society and the Electrochemical Society.

Selected publications:

Palacin, S.; Chin, D. N.; Simanek, E. E.; MacDonald, J. C.; Whitesides, G. M.; McBride, M. T.; Palmore, G. T. R. "Hydrogen-Bonded Tapes Based on Symmetrically Substituted Diketopiperazines: A Robust Structural Motif for the Engineering of Molecular Solids" J. Am. Chem. Soc. 1997, in press.

Palmore, G. T. R.; McBride, M. T. "Engineering Layers in Molecular Solids with the Cyclic Dipeptide of S-Aspartic Acid" Chem. Comm. 1997, in press.

Palmore, G. T. R.; McBride, M. T. "Pyridinium-Trifluoroacetate: Spoked Columns of Hydrogen-Bonded Cyclic Dimers" Acta Cryst. 1997, C53, in press.

Palmore, G. T. R.; Smith, D. K.; Wrighton, M. S. "pH-Dependent Rectification in Redox Polymers: Characterization of Electrode-Confined Siloxane Polymers Containing Naphthoquinone and Benzylviologen Subunits" J. Phys. Chem. 1997, 101, 2437.

Simanek, E. E.; Tsoi, A.; Wang, C. C.; Whitesides, G. M.; McBride, M. T.; Palmore, G. T. R. "Benzimidazolene-2-thiones: A New Class of Molecules for the Engineering of Molecular Tapes in the Organic Solid State" Chem. Mater. 1997, 9, 1954.

\section{W.D. Wilson}

W. D. Wilson earned his Ph.D. in Theoretical Physics from The City University of New York in 1967, based on research performed on defects in alkali halides as a fellow at Brookhaven National Laboratory. Following a two-year post-doctoral appointment, where he worked and published with G. J. Dienes and Martin Blume, he left Brookhaven to join Sandia National Laboratories in 1969. He was a visiting scientist with the Harwell Theoretical Physics group (England) during the summer of 1973. In 1974, he was promoted to head the Theoretical Division, a post he held for ten years while conducting research in a number of areas including the behavior of gases in metals, first-wall fusion interactions and nuclear stopping power in solids. This work on the mobility and trapping of helium in solids uncovered the underlying atomistics of bubble formation in irradiated and tritiated solids, leading to a book chapter and a number of invited talks, notably in Europe. He earned the DOE Award for Outstanding Sustained Research in 1983. In 1984, he was promoted to head the Computations Department, a position which included supercomputer operations, communications, networking and numerical analysis divisions. He chaired the joint LLNL/LANL/SNL study on the use of Supercomputers in Nuclear Weapons Design and later chaired the National Academy of Sciences Panel on the Impact of Supercomputing in Materials Science in the U. S. He was granted a DOE award for The Research, Development and Testing Five-Year Plan in 1987. In 1990, he founded the Center for Computational Engineering at Sandia aimed at bringing together a multi-disciplinary scientific effort utilizing massively parallel processing as a tool; he chaired a National Academy of Sciences subcommittee on Information Technology in 1993. He has authored over 50 publications in professional journals. Most recently, he has developed computational methods for coupling the Schrödinger and Poisson-Boltzmann equations, enabling a first principles approach to understanding the electrochemical double layer surrounding a molecule including its electronic relaxation effects. $\mathrm{He}$ is presently chairman of the National Academy of Sciences Advanced Technology Oversite Committee

Selected publications:

W. D. Wilson and C. M. Schaldach, " $\mathrm{H}_{2} \mathrm{~S}$ and $\mathrm{HS}^{-}$Adsorption on a Charged $\mathrm{Cu}$ Surface in an Electrolyte: Effect of Ionic Strength", Proc. Mat. Res. Soc. Dec. 1997.

W. D. Wilson and C. M. Schaldach, "Cl- Adsorption on an $\mathrm{Al}_{2} \mathrm{O}_{3}$ Surface in an Electrolyte", Proc. Electrochem. Soc. Mtg., Montreal, Canada, May 4-9, 1997. (Electrochemical Society, Inc., Pennington, NJ, 1997, p. 481).

W. D. Wilson, C. M. Schaldach and W L. Bourcier, "Single- and double-layer Coupling of Schrödinger and Poisson-Boltzmann Equations", Chem. Phys. Lett., 267, 431 (1997).

W. D. Wilson, C. L. Bisson and C. M Schaldach, "Polarization-Induced Atomic Reconfigurations of Glycine on a Metal Surface", J. Colloid and Interface Science, 187, 201(1997). 


\section{References}

1 Tersoff, J., TMS Abstracts, 1996 TMS annual meeting, Anaheim, Feb. 4-8, 1996, B104 (1996).

2 Russel, V.A., Evans, C.C., Li, Wenjie, Ward, M.D., Science, 276, 575 (1997).

3 Stupp, S.I., LeBonheur, V., Walker, K, Li, S.I., Huggins, K.E., Keser, M., and Amstutz, A., Science 276, 384 (1997).

4 Zerkowski, J.A., Seto, C.T., Wierda, D.A., Whitesides, G.M., J. Am. Chem. Soc., 1990, 112, 9025.

5 Mann, S. (1993). Nature, 365, p. 499-505.

6 Berman, A., Hanson, J., Leiserowitz, L., Koetzle, T. F., Weiner, S., and Addadi, L., Science 259, 776 (1993).

7 Mann, S., Archibald, D. D., Didymus, J. M., Douglas, T., Heywood, B. R., Meldrum, F. C., and Reeves, N.J. Science 261, 1286 (1993).

8 Belcher, A.M., Wu, X.H., Christensen, R.J., Hansma, P.K., Stucky, G.D. and Morse, D.E., Nature, 381, 56 (1996).

9 Addadi, L, Moradian, J., Shay, E., Maroudas, N.G. and Weiner, S., Proc. Nat. Acad. Sci., 84, 2732 (1987).

10 See for example: Demczyk, B.G., Garfunkel, E., Clemens, B.M, Williams, E.D. and Cuomo, J.J., ed., Evolution of thin film and surface morphology, Mat. Res. Soc. Symp. Proc. 355, (1995).

11 McPherson, A., Malkin, A.J. and Kuznetsov, Y.G. Ways and Means 3, 759 (1995).

12 Gratz, A.J., Hillner, P.E. and Hansma, P.K. Geochim. Cosmochim. Acta, 57, 491 (1993).

13 Land, T.A., De Yoreo, J.J., Lee, J.D. and Ferguson, J.R., In B.G. Demczyk et al., ed., Evolution of thin film and surface morphology, Mat. Res. Soc. Symp. Proc. , 355,45 (1995).

14 Malkin, T.A., Kutznesov, Yu. G., Land, T.A., De Yoreo, J.J. and McPherson, A. Nature Structural Biol., 2, 956 (1995).

15 Land, T.A., De Yoreo, J.J., Lee, J.D., Malkin, A.J., Kutznesov, Yu. G. and McPhersun, A.Surf. Sci. 384, 136 (1997).

16 Mc Bride, M., Land, T.A., Palmore, G.T.R. and De Yoreo, J.J., (in prep).

17 Mann, S., Nature, 332, 119 (1988).

18 Palacin, S., Chin, D.N., Simanek, E.E., MacDonald,J.C., Whitesides, G.M., McBride, M.T., Palmore, G.T.R., J. Am. Chem. Soc., (in press).

19 Crenshaw, M.A., In Skeletal Biomineralization: Patterns, Processes and Evolutionary Trends. Ed. J.G. Carter, Van Nostrand, New York., 1990, p. 1-9.

20 Didymus, J.M., Mann, S. , Sanderson, N.P., Oliver, P.; Heywood, B.R. and Aso-Samper, E.J., In Mechanisms and Phylogeny of Calcification in Biological Systems, Eds. S. Suga and H. Nakara, Springer-Verlag, 267 (1991).

21 Collins. M. J., Westbroek, P., Muyzer, G., DeLeeuw, J.W., Geochim. Cosmochim. Acta, 56, 1539 (1992).

22 Berman, A., Addadi, L., and Weiner, S., Nature 331, 546 (1988).

23 Sikes, C.S., M.L. Yeung and A.P. Wheeler, In Surface Reactive Peptides and Polymers: Discovery and

Commercialization. C.S. Sikes and A.P. Wheeler, Eds. (ACS Books: Washington, 1990) ch. 5.

24 Fink, D.J., Arnold, I.C. and Heuer, A.H., MRS Bulletin, XVII, 27 (1992).

25 Calvert, P., Biomimetic ceramics and composites, MRS Bulletin, XVII, 37 (1992).

26 Berman, A., Ahn, D. J., Lio, A., Salmeron, M., Reichert, A., and Charych, D., Science 259, 515 (1995).

27 Archibald, D. D., Qadri, S. B., and Gaber B. P., Langmuir 12, 538 (1996).

28 Heywood, B.R. and Mann, S., Langmuir, 8, 538 (1992).

29 Teng, H., Orme, C., Dove., P.M., and De Yoreo, J.J. (in prep).

30 Paquette, J. and Reeder, R.J., Geochim. et Cosmochim. Acta 59, 735 (1995).

31 Burton, W.K., N. Carbrera and F.C. Frank, Royal Soc. London Philos. Trans., A243, 299 (1951).

32 Chernov, A.A., Soviet Phys., 4, 116 (1961).

33 Gilmer, G.H., Ghez, R. and Cabrera, N., J. Cryst. Growth, 8, 79 (1971).

34 Theis, W. and Tromp, R.M., Phys. Rev. Lett. 76, 15, 2770 (1996).

35 Rashkovich, L.N. KDP Family of Crystals, Adam-Hilger, New York (1991).

36 Vekilov, P.G., Kuznetsov, Yu.G. and Chernov, A.A., J. Cryst. Growth, 121, 643 (1992).

37 De Yoreo, J.J., Land, and Lee, J.D., Phys. Rev. Lett. , 78, 4462 (1997).

38 Malkin, A.J., Kuznetsov, Yu. G., Glantz, W. and McPherson, A., J. Phys. Chem., 100, 11736 (1996).

39 Bales, G.S. and Chrzan, D.C., Phys. Rev. B , 506057 (1994).

40 Shitara, T., Vvedensky, D.D., Wilby, M.R., Z'hang, J., Neave, J.H., and Joyce, B.A., Phys. Rev. B , 46, 6815 (1992).

41 Orme, C. and Orr, B.G. , Surf. Rev. and Let. 471 (1997).

42 Krug,J. and Spohn, H., Europhys. Lett. , 8, 219 (1989).

43 Gilmer, G.H., J. Cry Growth, 35, 15 (1976).

44 McCoy,J.M. and LaFemina, J.P, Surface Science ,373, 288 (1997).

45 Maksym,P.A., Semiconductor Sci.Technol. , 3, 594 (1988).

46 de Leeuw, N. H. and Parker, S.C., J.Chem. Soc.Faraday Trans. , 93, 467 (1997).

47 Glosli, J.N. and Philpott, M. R., Electrochimica Acta 41, 2145 (1996).

48 John Harding, University College London, private communication.

49 For example, F.H. Stillinger and A. Rahman, J. Chem. Phys. 60, 1545 (1974), O. Steinhauser, Mol. Phys. 45,335 (1982), Jorgensen W.L., Chandrasekhar J., Madura J.D., Impey R. W., Klein M.L., J. Chem. Phys. 79, 926 (1985), Berendsen H.J., Grigera J.R., Straatsma T.P., et al., J. Phys. Chem. 91, 6269 (1987).

50 Wilson, W. D., Schaldach, C. M. and Bourcier, W. L., Chem. Phys. Lett. 267,431(1997).

51 Wilson, W. D. and Schaldach, C. M., Proc. Electrochem. Soc. Meeting, Montreal, Canada, May 4-9,1997 (Vol. 97-7, Electrochem. Soc., Inc. ,Pennington, N.J.(1997),p.481.

52 Wilson, W. D., Bisson, C. L. and Schaldach, C. M., J. Colloid and Interface Science, 187, 201 (1997).

53 Mayo, S. L., Olafson, B. D. and Goddard, W. A III, J. Phys. Chem. 94, 8897 (1990). 


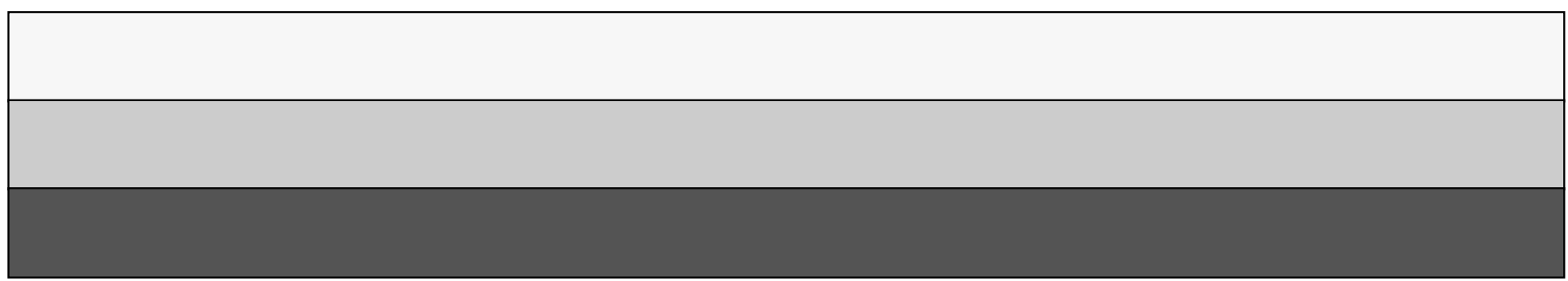

